\title{
Using Feynman's Tree Theorem to Evaluate Loop Integrals Numerically
}

\section{Wolfgang Kilian}

University of Siegen, Fachbereich Physik

E-mail: kilian@physik.uni-siegen.de

\section{Tobias Kleinschmidt* ${ }^{*}$}

University of Durham, Institute for Particle Physics Phenomenology

E-mail: tobias.kleinschmidt@durham.ac.uk

\begin{abstract}
We report on a new method for the numerical evaluation of loop integrals, based on the Feynman Tree Theorem. The loop integrals are replaced by phase-space integration over fictitious extra on-shell particles. This integration can be performed alongside with the Monte-Carlo integration of ordinary phase space, directly leading to NLO event generation.
\end{abstract}

RADCOR 2009 - 9th International Symposium on Radiative Corrections (Applications of Quantum Field Theory to Phenomenology)

October 25-30 2009

Ascona, Switzerland

*Speaker.

${ }^{\dagger}$ Supported by the UK Science and Technology Facilities Council (STFC), PP/E 0073717/1. 


\section{Introduction}

A crucial problem of event generation at next-to-leading order (NLO) in perturbation theory originates from the loop contributions to multi-parton matrix elements. The number of individual Feynman graphs rises dramatically with the number of external legs, and tensor reduction methods increase the number of terms even more. This poor scaling behavior, and the associated instabilities due to large numerical cancellations in matrix elements, make it worthwhile to investigate different and alternative approaches to the problem of automatic NLO computation and simulation.

We propose a new method for the evaluation of loop integrals, which allows for direct numerical computation without a decomposition into a set of basic integrals and corresponding large coefficients. ${ }^{1}$ These proceedings summarize the work of a recent publication [2]. Here, the matrix element is re-expressed using an improved version of the Feynman Tree Theorem (FTT) [3]. The loop integrals are transformed into phase space integrals and are evaluated numerically along with the phase space integral over the external partons of the process under consideration. Thus, there is only one step of numerical integration involved in the computation of any particular integrated cross section or distribution. We therefore can make use of powerful existing technologies for numerical phase-space integration by tree-level event generators to evaluate NLO processes.

\section{Feynman Tree Theorem}

The integrand $I(k)$ of a loop integral over momentum $k$ can be written as a product of Feynman propagators $F$ times a regular function $N(k)$ in the numerator. Suppressing possible indices in the numerator, we have

$$
I(k)=N(k) \prod_{i} F\left(k+p_{i}, m_{i}\right) .
$$

The $p_{i}$ are linear combinations of external momenta, $m_{i}$ the masses of the physical particle the propagator corresponds to. We define

$$
F_{i} \equiv F\left(k+p_{i}, m_{i}\right)=\frac{i}{\left(k+p_{i}\right)^{2}-m_{i}^{2}+i \varepsilon},
$$

using 't Hooft-Feynman gauge throughout this discussion.

The Feynman Tree Theorem [3] states, that the loop integral over (2.1) can be replaced by an integral over a sum of terms, where in each term one or more propagators $F_{i}$ are replaced by $\delta$-functions,

$$
\int d k \cdot I(k)=\int d k \cdot N(k)\left[\sum \Delta^{l} F \cdots-\sum \Delta^{l} \Delta^{l} F \cdots+\ldots-(-1)^{n} \sum \Delta^{l} \cdots \Delta^{l}\right] .
$$

with $d k \equiv d^{4} k \cdot(2 \pi)^{-4}$. The $\delta$-functions $\Delta_{i}^{l}=\frac{2 \pi}{2 E_{i}} \delta\left(k^{0}-\left(-p_{i}^{0}+E_{i}\right)\right)$ cancel the $k^{0}$-integration and cut the loop by setting the momentum of the original propagator $F_{i}$ on its mass-shell. The numerator structure of this propagator factorizes into a product of wave functions of the corresponding particle. The original loop integral can therefore be written as a sum of tree graphs, with a phase space integration replacing the loop integral.

\footnotetext{
${ }^{1}$ This is in contrast to methods used for recent computations of $2 \rightarrow 4$ processes at the LHC [1]. These either use Passarino-Veltman style reductions or a unitarity based approach to decompose amplitudes into a set of basic integrals.
} 
Derivations of the FTT in its form (2.3) can also be found in [4] and [5]. In the latter work, Catani et al. exploited the relation between loop integrals and phase space integrals to express loop amplitudes as sum of tree amplitudes arising solely from single cuts. Contributions from multiple cuts, which are present in (2.3), are compensated by a non-trivial $i \varepsilon$-prescription in the propagators.

To make use of the FTT in a numerical evaluation of loop integrals involving only real numbers, at some point we have to set the $i \varepsilon$ terms in the denominators to zero. Using the identity

$$
\frac{1}{x-a \pm i \varepsilon}=\mathscr{P} \frac{1}{x-a} \mp i \pi \delta(x-a)
$$

where $\mathscr{P}$ is Cauchy's Principal Value, we can rewrite (2.2) after a partial fraction decomposition:

$$
F_{i}=P_{i}+\frac{1}{2} \Delta_{i}^{l}+\frac{1}{2} \Delta_{i}^{u}
$$

where we defined $\Delta_{i}^{u}=\frac{2 \pi}{2 E_{i}} \delta\left(k^{0}-\left(-p_{i}^{0}-E_{i}\right)\right)$, as the delta function that sets the associated fourmomentum on its mass-shell with negative zero component. $P_{i}$ stands for the propagator with no $i \varepsilon$-prescription in the denominator.

Replacing the propagators $F_{i}$ in (2.3) by (2.5), we obtain a version of the FTT, which is better suited for a direct numerical integration:

$$
\begin{aligned}
\int d k \cdot I(k)= & \int d k \cdot N(k)\left[\Delta_{1}^{l} P_{2} \cdots P_{n}+P_{1} \Delta_{2}^{l} P_{3} \cdots P_{n}+\ldots+P_{1} \cdots P_{n-1} \Delta_{n}^{l}\right] \\
& +\int d k \cdot N(k) \sum_{\substack{\text { perm } \\
U+L \geq 2}} \frac{1}{2^{L+U}}\left(1-(-1)^{L}\right) \Delta^{l^{L}} \Delta^{u^{U}} P^{P}
\end{aligned}
$$

The sum runs over all possible permutations, where the functions $\left(\Delta^{l}, \Delta^{u}, P\right)$ appear $(L, U, P)$ times.

We explicitly wrote out the terms containing one $\Delta^{l}$ function in the first line of (2.6). After $k^{0}$-integration, all of these terms can be interpreted as tree graphs with one additional incoming and outgoing particle and an additional phase space integral over this particles momentum. This integral can now be pulled out of the individual graphs and put in front of the amplitude, alongside the phase space integrals over the external particles which are present in a cross-section calculation. Since the extra integration is also of the form of a phase-space integral, techniques developed for the integration, in particular multi-channel sampling, can immediately be adopted. Furthermore, this integration can be performed simultaneously with the external phase space integration, which is in contrast to common analytical methods, where for each individual configuration of external momenta the analytical result of the loop integration has to be numerically evaluated.

If the momenta of two or more propagators go on-shell simultaneously in the momentum integration, terms with more than one $\Delta$-function in (2.6) are non-vanishing. These give rise to further contribution to the final result, but can be evaluated rather easily, since after the $k^{0}$-integration, the remaining $\delta$-functions lower the dimension of integration to two or less.

\section{UV and IR Subtraction}

As long as we are dealing with massive theories, a convenient set of renormalization conditions is given by the on-shell renormalization scheme. This requires the pole of the real part of 
propagators to coincide with the corresponding particle mass, with residue 1. Vertex functions are set equal to the tree level vertex functions for on-shell external legs.

We make use of a variation of the BPHZ procedure, which results in loop graphs acting as counterterms. These can be evaluated under the same phase space integral over the loop momentum. UV divergences are then cancelled locally in three-momentum space.

Consider a 1PI one-loop graph $\Gamma^{n}\left(p_{1}, \ldots, p_{n}\right)$ with superficial degree of divergence $\omega(\Gamma)$. We define the T operator as a Taylor expansion around on-shell momenta $\bar{p}_{i}$, with $\bar{p}_{i}^{2}=m_{i}^{2}$ :

$$
\begin{aligned}
T \circ \Gamma^{n}\left(p_{1}, \ldots, p_{n}\right)= & \Gamma^{n}\left(\bar{p}_{1}, \ldots, \bar{p}_{n}\right)+\left.\sum_{i}^{n-1}\left(p_{i}-\bar{p}_{i}\right)^{\mu} \frac{\partial \Gamma^{n}}{\partial p_{i}^{\mu}}\right|_{p_{1}=\bar{p}_{1}, \ldots, p_{n}=\bar{p}_{n}}+ \\
& \ldots+ \\
& \left.\frac{1}{d !} \sum_{i_{1}, \ldots, i_{d}}^{n-1}\left(p_{i_{1}}-\bar{p}_{i_{1}}\right)^{\mu_{1}} \ldots\left(p_{i_{d}}-\bar{p}_{i_{d}}\right)^{\mu_{d}} \frac{\partial^{d} \Gamma^{n}}{\partial p_{i_{1}}^{\mu_{1}} \ldots \partial p_{i_{d}}^{\mu_{d}}}\right|_{p_{1}=\bar{p}_{1}, \ldots, p_{n}=\bar{p}_{n}},
\end{aligned}
$$

up to $d=\omega(\Gamma)$. With this T operator, the renormalized 1PI n-point functions

$$
\hat{\Gamma}^{n}\left(p_{1}, \ldots, p_{n}\right)=\Gamma^{n}\left(p_{1}, \ldots, p_{n}\right)-T \circ \Gamma^{n}\left(p_{1}, \ldots, p_{n}\right)
$$

fulfill the renormalization conditions of the on-shell scheme.

The expressions resulting from (3.1) can also be interpreted as loop graphs and are easily derived from the original Feynman graph. Applying again the FTT on the subtraction graphs, the resulting phase space integral over all tree graphs is UV finite.

A closer inspection of the tree graphs of the FTT reveals, that the IR divergent parts solely arise from terms with a cut massless propagator. These can be immediately related to the corresponding IR divergent real emission graph by crossing the incoming piece of the cut propagator.

Although in the limit of zero momentum-flow through this particle, the two contributions cancel each other, a suitable projection of the real emission part onto the virtual part is needed to cancel the IR peak locally in three-momentum space. For soft divergences, a simple approximation is obtained by setting the two contributions equal for momenta lower than a given soft energy $E_{s}$.

In the light of collinear divergences, a more sophisticated ansatz is to construct dipole subtractions, which cancel IR divergences locally also on the virtual side. The sum of the resulting analytic integrals for both parts of the dipoles would then be finite. This is left for future works.

\section{Threshold Singularities}

When the momentum integration is performed in the first line of (2.6), the integrand might get peaks in parts of the phase space where momenta of un-cut propagators are on-shell. The occurrence of such peaks, although analytically integrable, leads to problems in the numerical evaluation of the integrand. In a series of papers for a direct numerical integration of massless amplitudes, Soper et al. [6] proposed a contour deformation of the integration into the complex plane to avoid the singularities. To allow for a direct implementation in an ordinary Monte Carlo generator, we add subtraction terms with zero real value but with the same peak structure to the integrand. These will cancel the peaks and allow for a better convergence in the numerical evaluation. 

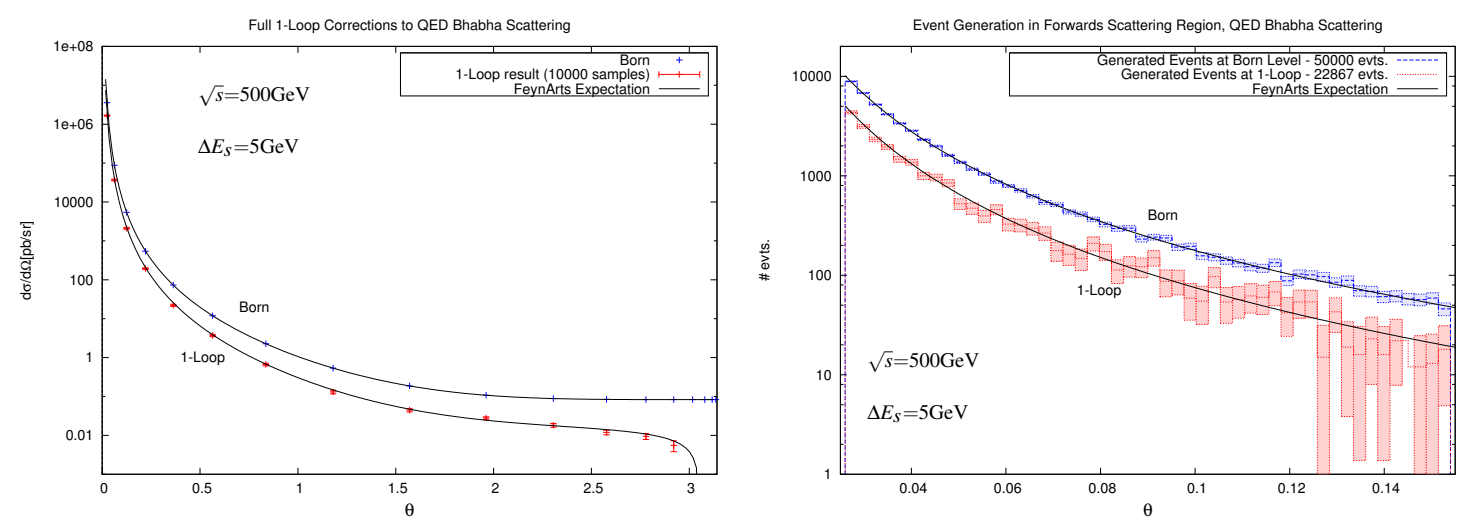

Figure 1: Differential cross sections for Monte Carlo integration and event generation of Bhabha scattering.

In the sum of the tree graphs, peaks remain if $p_{j i}^{2} \equiv\left(p_{i}-p_{j}\right)^{2}>\left(m_{i}+m_{j}\right)^{2}$. The momentum constellation is such, that the two real particles could be produced at the same time. In the rest frame of $p_{j i}^{2}$, the peak of the threshold singularity is spherical with radius $\mathbf{k}_{s}$ and a simple pole

$$
I(\mathbf{k}) \propto\left(\mathbf{k}-\mathbf{k}_{s}\right)^{-1}, \quad \quad \mathbf{k}_{s}=\lambda^{\frac{1}{2}}\left(p_{j i}^{02}, m_{i}^{2}, m_{j}^{2}\right) \cdot\left(2\left|p_{j i}^{0}\right|\right)^{-1},
$$

$\lambda$ being the Källén function. We can now evaluate the residue of the integrand and divide it by the singular structure. Adding the resulting function to the integrand symmetrically around the spherical peak, the peak will be cancelled and the integrated contribution of the fix function vanishes. More precisely, after a Lorentz transformation into the rest frame of $p_{j i}^{2}$, the function we use reads

$$
\mathrm{F}\left(\mathbf{k}^{\prime}\right) \equiv \frac{\mathbf{k}_{s} R\left(\Lambda^{-1} k_{s}^{\prime}-p_{i}\right)}{4 p_{j i}^{0}}\left(\frac{1}{\mathbf{k}^{\prime}-\mathbf{k}_{s}}-2 \frac{\mathbf{k}^{\prime}-\mathbf{k}_{s}}{c^{2}}+\frac{\left(\mathbf{k}^{\prime}-\mathbf{k}_{s}\right)^{3}}{c^{4}}\right) \theta\left(\mathbf{k}^{\prime}-\left(\mathbf{k}_{s}-c\right)\right) \theta\left(\left(\mathbf{k}_{s}+c\right)-\mathbf{k}^{\prime}\right) .
$$

This has to be transformed back to the integration frame and added to the integrand. Here, we also added a linear and cubic term in $\left(\mathbf{k}^{\prime}-\mathbf{k}_{s}\right)$, to make the joined integrand continuous and differentiable at the artificial borders introduced by the theta functions.

It is possible, that for higher n-point functions there are overlapping threshold singularities. In this case, we can add further fix functions similar to (4.2) to cancel the peak structure. Since in this case, some of the added functions do not vanish after integration and give a small contribution to the final result, the width parameter $c$ in (4.2) should be taken considerably small. This leads to a trade-off between accuracy and efficiency in the final integration.

\section{Results}

As a first application, we evaluate the one-loop cross section of Bhabha scattering in massive QED. The Bhabha scattering process is ideally suited to demonstrate the evaluation of processes at NLO by the Feynman Tree Theorem. The one-loop result for the cross section is well known and can easily be produced using automated loop-graph evaluation tools. Most complications inherent in the method - UV subtractions, IR cancellations, and threshold singularities - are present simultaneously. There are ten graphs in the one-loop corrections, which after rewriting them as tree graphs lead to a rich structure in the integrand. This has to be treated by multichannel integration methods. Furthermore, the smallness of the electron mass compared to the energy of $500 \mathrm{GeV}$ where we evaluate the process provides a stringent test of the stability of the numerical integration. 
We created analytical expressions for the loop graphs in computer-readable form using the Mathematica- and FORM-based packages FeynArts and FormCalc [7]. The matrix elements were then handed over to a specially crafted Mathematica program that creates subtraction graphs, cuts the loops and, where needed, calculates the fixing functions. For each tree graph, the program creates parameterizations (integration channels) which map the resulting phase space onto the unit hypercube, taking into account the peak structure. The resulting expressions for the matrix elements and the channels are then written out in Fortran code.

As an integration routine, we choose the multi-channel algorithm VAMP [8]. We compare the final results for the cross section and angular distribution to an independent calculation that proceeds along the usual way of integration via tensor reduction, using FeynArts and FormCalc. In the left plot of figure 1, we show the differential cross section for the full matrix element. The results are in complete agreement with FeynArts. The adaption of the grids in the multi-channel approach to the peaks works quite efficient. With 10000 sampling points, the error estimate on the numerical integration as returned by the Monte-Carlo integrator is less than $1 \%$.

The right hand plot of figure 1 shows results for event generation of the partonic QED process in the forwards scattering region. The distribution of events is again in agreement with predictions from FeynArts. Using the integrand obtained from the FTT, we generate unweighted events with a phase space including the on-shell momentum of a loop particle. This increases the dimension of integration from one to four. The efficiency of event generation is in the percent-regime.

\section{Conclusions}

We reported on a new method for computing NLO corrections to scattering cross sections. Here, all integrals are transformed into ordinary phase-space integrals (albeit with unusual boundaries) that can be handled by an ordinary numerical multi-channel phase-space integrator. To this hand, we had not just to implement subtractions for UV and IR singularities, but furthermore subtraction functions (fixing functions) for threshold singularities which do not cause problems in the usual semi-analytic methods.

Extending the method to the full Standard Model and multi-particle processes, it promises important advantages over more conventional semi-analytic algorithms: The computational complexity does not increase dramatically with the number of legs in loop diagrams. Evaluating a NLO $n$-particle process should require similar CPU resources as a LO $n+1$-particle process, summed inclusively over all particle species. Combining loop integration and phase-space sampling in a single step, we avoid a whole layer in the calculation. In particular, all terms are evaluated only up to the level of precision that is required by the actual simulation.

There is still a long way before this method can actually improve the simulation of physics processes in the Standard Model or its extensions. On the one hand, we have to handle the more complicated IR behavior of QCD and state suitable renormalization conditions for the non-abelian theory. On the other hand, the method has to be augmented by a consistent treatment of unstable states, which appear in loops and, in our case, would become artificial external particles in event samples. Nevertheless, the method, has distinct advantages that warrant its further development towards realistic complete LHC and ILC applications. 


\section{References}

[1] A. Bredenstein, A. Denner, S. Dittmaier and S. Pozzorini, Phys. Rev. Lett. 103 (2009) 012002 [arXiv:0905.0110 [hep-ph]]; G. Bevilacqua, M. Czakon, C. G. Papadopoulos, R. Pittau and M. Worek, JHEP 0909 (2009) 109 [arXiv:0907.4723 [hep-ph]]; A. van Hameren, C. G. Papadopoulos and R. Pittau, JHEP 0909 (2009) 106 [arXiv:0903.4665 [hep-ph]]; C. F. Berger et al., arXiv:0907.1984 [hep-ph]; C. F. Berger et al., Phys. Rev. Lett. 102 (2009) 222001 [arXiv:0902.2760 [hep-ph]]; R. K. Ellis, W. T. Giele, Z. Kunszt, K. Melnikov and G. Zanderighi, JHEP 0901 (2009) 012 [arXiv:0810.2762 [hep-ph]]; K. Melnikov and G. Zanderighi, arXiv:0910.3671 [hep-ph]; T. Binoth et al., arXiv:0807.0605 [hep-ph]; T. Reiter, arXiv:0903.0947 [hep-ph];

[2] W. Kilian and T. Kleinschmidt, arXiv:0912.3495 [hep-ph].

[3] R. P. Feynman, Acta Phys. Polon. 24, 697 (1963); R. P. Feynman, In *J R Klauder, Magic Without Magic*, San Francisco 1972, 355-375; R. P. Feynman, In *J R Klauder, Magic Without Magic*, San Francisco 1972, 377-408.

[4] A. Brandhuber, B. Spence and G. Travaglini, JHEP 0601, 142 (2006) [arXiv:hep-th/0510253].

[5] S. Catani, T. Gleisberg, F. Krauss, G. Rodrigo and J. C. Winter, JHEP 0809, 065 (2008) [arXiv:0804.3170 [hep-ph]].

[6] D. E. Soper, Phys. Rev. Lett. 81, 2638 (1998) [arXiv:hep-ph/9804454]; D. E. Soper, Phys. Rev. D 62 (2000) 014009 [arXiv:hep-ph/9910292]; M. Krämer and D. E. Soper, Phys. Rev. D 66 (2002) 054017 [arXiv:hep-ph/0204113]; Z. Nagy and D. E. Soper, Phys. Rev. D 74 (2006) 093006 [arXiv:hep-ph/0610028].

[7] T. Hahn, Comput. Phys. Commun. 140, 418 (2001) [arXiv:hep-ph/0012260]; T. Hahn and M. Perez-Victoria, Comput. Phys. Commun. 118, 153 (1999) [arXiv:hep-ph/9807565].

[8] T. Ohl, Comput. Phys. Commun. 120, 13 (1999) [arXiv:hep-ph/9806432]. 